
internationales

vol. 20 - n² 2004

L'asile politique en Europe depuis l'entre-deux-guerres

\title{
Les réfugiés palestiniens en Europe. Complexité des parcours et des espaces migratoires
}

\section{Mohamed Kamel Doraï}

\section{(2) OpenEdition \\ 1 Journals}

Édition électronique

URL : https://journals.openedition.org/remi/986

DOI : $10.4000 /$ remi.986

ISSN : $1777-5418$

Éditeur

Université de Poitiers

\section{Édition imprimée}

Date de publication : 1 septembre 2004

Pagination : 169-186

ISBN : 2-911627-37-7

ISSN : 0765-0752

Référence électronique

Mohamed Kamel Doraï, « Les réfugiés palestiniens en Europe. Complexité des parcours et des espaces migratoires », Revue européenne des migrations internationales [En ligne], vol. 20 - n² | 2004, mis en ligne le 25 septembre 2008, consulté le 15 avril 2022. URL : http://journals.openedition.org/ remi/986 ; DOI : https://doi.org/10.4000/remi.986

Ce document a été généré automatiquement le 15 avril 2022.

(c) Université de Poitiers 


\title{
Les réfugiés palestiniens en Europe. Complexité des parcours et des espaces migratoires
}

\author{
Mohamed Kamel Doraï
}

\section{NOTE DE L'ÉDITEUR}

Ce texte a été publié une première fois dans Guillon, Legoux, Ma Mung éds, 2003.

1 Les Palestiniens qui résident en Europe forment une communauté récente, dont la majeure partie des membres est arrivée dans les années quatre-vingt. Même si leur nombre est assez peu important, puisqu'il y a certainement moins de 100000 réfugiés palestiniens en Europe, leur situation est intéressante à différents titres.

De façon générale, les réfugiés ont trois possibilités en ce qui concerne leur avenir : le retour dans leur pays d'origine, l'installation dans leur pays de résidence ou la réinstallation dans un pays tiers. Le droit au retour des réfugiés est l'une des principales revendications de l'ensemble des Palestiniens, où qu'ils résident. Il s'appuie sur la résolution 194 de l'Assemblée générale des Nations Unies votée en 1948. Cependant, la situation politique actuelle et l'état d'avancée du processus de paix n'ont pas encore envisagé cette question. L'incertitude pour l'avenir, ainsi que les guerres qui se déroulent au Moyen-Orient, ont poussé un certain nombre de réfugiés palestiniens à un nouvel exil vers des pays à l'extérieur du monde arabe.

3 L'Europe s'est présentée à partir des années soixante-dix comme un espace favorable à la venue de Palestiniens et cela pour deux raisons principales: la possibilité pour certains d'y obtenir l'asile politique, et l'opportunité pour tous d'y trouver du travail et d'assurer à leur famille une vie plus stable et un avenir plus sûr pour leurs enfants. L'Europe peut-elle devenir dans l'avenir une région importante au sein de la diaspora palestinienne? S'agit-il d'une région de réinstallation des Palestiniens ou bien d'un espace d'émigration de travail comme les pays producteurs de pétrole? Peut-on 
imaginer une évolution équivalente à celle des Palestiniens des États-Unis qui forment un groupe dynamique et structuré au sein de la diaspora? Après un panorama général des principales communautés palestiniennes en Europe, la situation juridique des Palestiniens du Liban, principale cause de départ des réfugiés est précisée; puis les principales phases d'arrivée en Europe sont distinguées pour ensuite analyser le fonctionnement actuel de la migration clandestine. La situation en Suède est décrite plus amplement, du fait des spécificités de ce pays d'accueil et la relative méconnaissance de la situation des Palestiniens qui y résident. Ce travail se fonde essentiellement sur deux études de terrain au Liban et en Suède.

\section{Les Palestiniens en Europe : situation générale}

Le contexte juridique difficile au Moyen-Orient, ainsi que les conflits qui ont marqué le Liban de 1975 à 1991 ont poussé les réfugiés palestiniens à émigrer pour trouver du travail, assurer leur avenir ou fuir les combats. Ils se sont dirigés vers les pays du Golfe, comme nombre de leurs homologues de Jordanie, mais aussi vers l'Europe du Nord, et plus particulièrement l'Allemagne, la Suède, le Danemark et le Royaume-Uni.

\section{Allemagne}

5 Il est difficile de définir le nombre de Palestiniens qui résident en Allemagne, aucune donnée statistique n'étant effectivement disponible. Elle semble cependant être la plus importante communauté palestinienne en Europe, dont on peut estimer les effectifs à environ 30000 personnes et aussi une des plus anciennes. Ce pays a été choisi parce qu'il était aisé d'y entrer par Berlin-Est, et l'asile politique était lui aussi assez facile à obtenir dans les années soixante-dix à Berlin-Ouest. La situation de l'emploi y était aussi favorable à la même époque. La majeure partie des Palestiniens résident actuellement à Berlin ainsi que dans les grandes villes allemandes. L'Allemagne a fermé ses portes à l'entrée des Palestiniens vers la fin des années quatre-vingt. D'un pays d'accueil, elle est alors devenue un pays de transit vers l'Europe du Nord.

\section{Suède}

6 Le nombre de Palestiniens en Suède est estimé à 13000 par le Bureau de l'immigration suédois en 1998. Ce chiffre reste cependant une estimation, la Suède n'enregistrant pas les réfugiés palestiniens parce qu'ils ne disposent pas d'une nationalité reconnue. En l'absence de nationalité, ils sont classés comme apatrides ou de nationalité inconnue. Le Bureau de l'immigration indique que la quasi-totalité des apatrides qu'il recense sont des Palestiniens. Les différents entretiens que nous avons pu effectuer en Suède nous permettent, pour notre part, d'estimer le nombre de Palestiniens entre 10000 et 15000 individus. Tout dépend de la prise en compte ou non de la deuxième génération qui est suédoise de naissance, issue de couples palestiniens d'origine ou de couples mixtes. De fait la quasi-totalité des Palestiniens disposent de la nationalité suédoise qui leur est accordée après un délai pouvant aller de trois à cinq ans, selon les situations que nous avons rencontrées. La répartition géographique des Palestiniens fait apparaître une concentration dans les principales agglomérations suédoises, et plus particulièrement 
Stockholm (environ 5 000), Uppsala (3 000), puis Malmö (1 500), Göteborg (1 500) et Norrköping qui concentre le reste de la population palestinienne.

\section{Danemark}

$7 \quad$ La situation des Palestiniens est au Danemark assez semblable à ce qu'elle est en Suède. La plupart d'entre eux ont obtenu l'asile dans ce pays à la suite de l'invasion israélienne de 1982 et de la guerre des camps (1985-1987). Une grande partie a acquis la nationalité danoise. L'intégration à la société danoise semble avoir été difficile et des tensions existent entre les deux communautés. Il est assez complexe de connaître le nombre de Palestiniens qui résident au Danemark, mais on peut estimer qu'il est sensiblement égal à celui de la Suède et peut donc être estimé entre 10000 et 20000.

\section{Royaume-Uni}

La Grande-Bretagne est une ancienne destination pour les Palestiniens. On y trouve surtout des étudiants et encore peu de travailleurs. On peut estimer que la communauté compte quelques milliers de personnes aujourd'hui. Cependant, les entretiens que nous avons réalisés au Liban nous permettent de dire que les réfugiés qui veulent s'y rendre sont de plus en plus nombreux, surtout en raison des facilités pour y trouver un emploi.

\section{La situation juridique précaire des Palestiniens au Liban, principal moteur de l'émigration}

9 À la suite des Accords de Taëf (1991) qui ont mis fin à la guerre civile, la situation politique et économique des Palestiniens du Liban s'est dégradée considérablement pour plusieurs raisons :

- - la recomposition politique de l'État libanais a marginalisé les Palestiniens ;

- l'OLP s'est recentrée sur les territoires occupés puis sur les territoires autonomes avec la mise en place du processus de paix ;

- la situation économique du Liban de l'après-guerre a creusé les inégalités sociales en laissant une partie de la population, dont les Palestiniens font partie, dans une situation très précaire ;

- - les secteurs d'activités des Palestiniens du Liban (manutention, bâtiment, salariat agricole) connaissent une forte concurrence avec une importante main-d'œuvre étrangère présente sur place ;

- - enfin la perception qu'ont les Palestiniens du Liban des Accords d'Oslo comme ne leur offrant aucune solution à moyen ou long terme, les pousse à se diriger vers l'Europe pour acquérir un statut juridique stable et reconnu.

10 Cette détérioration de la situation politique et socio-économique des Palestiniens du Liban a donné une impulsion à l'émigration vers l'Europe. Les facteurs juridiques sont, d'après les entretiens que nous avons pu réaliser, l'un des principaux moteurs de leur émigration. Il faut avoir à l'esprit que la situation des Palestiniens au Liban est à lire dans le cadre d'un État affecté par seize ans de guerre civile, par deux invasions israéliennes en 1978 et 1982, et la fin toute récente de l'occupation israélienne du Liban 
Sud. D'autres populations civiles libanaises, comme une partie des chiites, vivent aussi dans des conditions très difficiles.

Juridiquement, les Palestiniens ne sont pas considérés comme différents des autres étrangers. L'étranger est ainsi défini : "Toute personne naturelle ou juridique qui n'est pas un sujet libanais" (loi du 10 Juillet 1962, article 1). Dans les faits, leur situation est pourtant différente, car les autorités libanaises ont créé des organismes spécifiques chargés de gérer leur situation.

\section{Un droit au séjour ambigu}

La loi libanaise de 1962 précise que tout étranger qui entre au Liban doit détenir des papiers officiels avec un visa d'entrée. Les Palestiniens, qui résident sur le sol libanais depuis 1948, ne disposent pas de tels documents. Le ministre de l'Intérieur a donc décidé de régulariser la situation des étrangers résidant au Liban avant 1962. Les Palestiniens sont définis comme "des étrangers qui ne portent pas de documents de leur pays d'origine et qui résident au Liban avec des cartes de résidence délivrées par la Direction de la Sûreté Générale, ou des cartes d'identification délivrées par la Direction Générale du Département des Affaires des Réfugiés au Liban " (Al-Natour, 1996). À ce titre, ils sont considérés comme des étrangers avec un statut spécial. Cette situation "d'étranger particulier » va se poursuivre avec la signature de l'Accord du Caire en 1969, Cet Accord indique que la légitimité de résidence des Palestiniens au Liban découle de facto de leur présence sur le sol libanais au moment de sa signature, et leur accorde les droits de résidence et de travail. Ils échappent donc à la législation générale sur le droit de résidence des étrangers au Liban. Mais cet accord a été abrogé par le parlement libanais en 1987. Une nouvelle tentative de formaliser les relations entre Libanais et Palestiniens a été tentée en 1991, mais s'est soldée par un échec.

\section{Des entraves à la mobilité internationale}

13 Les Palestiniens bénéficient d'une totale liberté de mouvement à l'intérieur du Liban. Par contre leur mobilité internationale est fortement entravée ${ }^{1}$. Ceux qui désirent se rendre à l'étranger doivent demander un passeport au Département des Affaires des Réfugiés, qui émet son opinion et transmet le document à la Direction de la Sûreté Générale. Depuis 1980, ils ne sont plus exemptés de visas et de taxes pour la demande ou le renouvellement de passeport. De plus, les Palestiniens arrivés en 1948 au Liban doivent obtenir un visa de sortie et d'entrée du Liban, contre paiement d'une taxe (décret 478, 1995). Ce dernier décret a considérablement limité la liberté de circulation à l'étranger des Palestiniens du Liban. Effectivement, de très nombreux Palestiniens estimés à 100000 par Razqallah (1998) - se trouvaient à l'étranger au moment de la promulgation de ce décret, et rares sont ceux qui ont pu obtenir un visa leur permettant de rentrer au Liban. Selon des sources palestiniennes, 30000 Palestiniens auraient ainsi perdu leur droit de résidence au Liban depuis 1995 (The Jerusalem Report, 7 août $1997: 28)$. 


\section{Un droit au travail très discriminant} le droit au travail. En tout, selon des témoignages de chercheurs recueillis au Liban, une cinquantaine de professions (soixante-dix selon The Jerusalem Report, 7 août 1997 : 28) leur seraient interdites. La réciprocité de traitement, qui ne peut être appliquée pour les Palestiniens qui ne disposent pas d'État, contredit les principes d'accueil et de facilité de travail énoncés dans l'Accord du Caire en 1969. Les Palestiniens se voient interdire l'accès de plus en plus de secteurs d'activités, après la promulgation du décret 289/1 de 1982 et le décret 3/1 de 1993. Ceci concerne entre autres les professions libérales, comme avocat, médecin, ou pharmacien ou les métiers comme ingénieur. De plus ils n'ont pas droit à la protection sociale en vertu de la réciprocité de traitement même quand ils cotisent. Il faut noter qu'une partie de ces professions peuvent être exercées par les Palestiniens dans le cadre des activités de l'UNRWA, qui emploie une main-d'œuvre quasi exclusivement palestinienne. D'autres choisissent de travailler sans contrat de travail, avec toute la précarité que cela suppose.

\section{Un accès à la naturalisation très limité}

Dans le domaine de l'attribution de la nationalité, le Liban applique le droit du sang (patrilinéaire) et dans certains cas le droit du sang et du sol associés. Une femme palestinienne qui se marie officiellement au Liban avec un Libanais peut se voir octroyer la nationalité libanaise. La naturalisation est soumise à une décision de l'État libanais qui peut la refuser. Le Liban respecte la recommandation de la Ligue Arabe, qui stipule que les Palestiniens ne doivent pas acquérir une autre nationalité. Cependant, plusieurs dizaines de milliers d'entre eux, surtout des chrétiens, ont été naturalisés entre 1952 et 1964. Pendant la guerre civile, certains ont obtenu la nationalité libanaise en trafiquant des passeports (Destremau, 1993).

\section{Les principales phases de l'arrivée des Palestiniens en Europe}

17 Cette situation juridique, économique et politique peu favorable, à laquelle il faut ajouter la guerre civile libanaise ainsi que les invasions israéliennes, ont poussé les Palestiniens du Liban à émigrer de façon assez importante vers l'Europe. D'autres zones de départ, comme la Syrie ou les territoires occupés et autonomes palestiniens, sont aussi concernés. Le choix de l'Europe, selon les entretiens que nous avons réalisés, est rarement volontaire. Pour un certain nombre de Palestiniens la présence de proches a été un facteur décisif dans ce choix, pour d'autres, des organisations humanitaires les 
ont dirigés directement vers la Suède ou le Danemark. Pour la majorité d'entre eux, l'Europe est l'ultime étape d'un parcours migratoire complexe, composé de séjours brefs et d'expulsions dans plusieurs pays européens, liés à leur absence de statut et de nationalité, et leur impossibilité de retourner au Liban à cause de la guerre. Les pays d'Europe qui les acceptent comme résidents apparaissent alors comme des espaces refuge, qui, par leurs dispositions juridiques, leur permettent d'obtenir un droit d'installation sur le territoire européen. L'arrivée des Palestiniens en Europe qui a débuté dans les années soixante, s'est déroulée en quatre phases principales, qui se différencient par le nombre d'arrivées et le type de migration. Alors que jusque dans les années soixante-dix peu de Palestiniens sont arrivés en Europe et sont venus essentiellement pour travailler, pendant les années quatre-vingt et quatre-vingt dix le nombre d'arrivées a augmenté de façon importante et l'on trouve de plus en plus de réfugiés politiques et de Palestiniens qui vivent dans des situations très difficiles.

\section{L'arrivée de quelques étudiants dans les années soixante}

Cette première phase de la migration palestinienne concerne essentiellement la Suède (Doraï, 2000). Le premier groupe de Palestiniens, formé d'étudiants originaires de Jordanie, du Liban, de Syrie et de Cisjordanie, venus pour se former professionnellement, est arrivé en 1962 dans le cadre d'un programme de courte durée. La majorité d'entre eux est rentrée, seuls 40 sont restés en Suède (Assar, 1995). Un certain nombre d'entre eux se sont mariés avec des Palestiniennes lors de leurs retours périodiques dans leur pays de premier accueil. Malgré leur situation sociale assez privilégiée par rapport aux Palestiniens arrivés ultérieurement, ils éprouvent, pour certains d'entre eux, des difficultés d'intégration. Nous avons pu rencontrer un de ces Palestiniens à Göteborg. Ahmed ${ }^{2}$ a choisi, à la fin de ses études, de s'installer en Suède, essentiellement en raison des possibilités d'emploi sur place, et de l'importante différence de niveau de vie entre la Suède et la Syrie. Il est retourné plusieurs fois en Syrie, sans jamais s'y installer de nouveau. Il s'est ensuite marié à une Palestinienne de Syrie à Damas. Il a pu la faire venir en Suède par le biais du regroupement familial. Avec ses trois jeunes enfants, il a un projet de retour en Syrie, essentiellement, à son sens, en raison des difficultés d'intégration qu'il connaît, bien que toute sa famille possède la nationalité suédoise.

\section{Les premiers des travailleurs réfugiés arrivent dans les années soixante-dix}

19 Quelques centaines de travailleurs palestiniens sont venus en Suède dans le cadre d'accords entre l'UNRWA et des entreprises suédoises comme Volvo. Un certain nombre d'entre eux décident de rester en Suède, toujours en raison du différentiel de niveau de vie entre leur pays de premier accueil (comme le Liban, la Syrie ou les Territoires occupés) et la Suède et de la possibilité d'obtenir la nationalité suédoise. Ils font ensuite venir leur famille, dans le but d'une installation définitive. D'après les entretiens que nous avons réalisés, on trouve aussi des travailleurs migrants au profil plus classique, qui peuvent venir de Syrie ou du Liban. Leur départ d'un pays arabe est motivé par la volonté de trouver un emploi mais aussi par les problèmes administratifs liés à leur statut juridique précaire. Ces deux premiers groupes de Palestiniens, qui ne représentent que quelques centaines de personnes, sont essentiellement des migrants 
économiques, à la recherche d'un emploi, mais aussi d'une situation juridique plus favorable qui leur permette d'acquérir une autonomie de déplacement.

C'est durant cette même période que se sont développées les arrivées de Palestiniens en Allemagne, beaucoup plus nombreuses que celles qui se sont dirigées vers la Suède. Ce sont surtout des travailleurs migrants qui tentent de s'y installer parce que la situation économique est bonne et qu'elle leur permet de s'installer durablement dans ce pays. L'Allemagne est alors, pour les Palestiniens, l'un des pays européens dans lesquels il est le plus facile d'entrer et d'obtenir une carte de résidence.

\section{La principale vague d'arrivée : les réfugiés dans les années 1980}

Les principaux pays d'accueil durant cette phase sont la Suède et le Danemark (Figures 1 et 2). Les réfugiés arrivent principalement après l'invasion israélienne du Liban en 1982. Ce groupe, de loin le plus important numériquement, se différencie très nettement des Palestiniens arrivés lors des deux phases précédentes. Leur départ du Liban n'a pas fait l'objet d'un choix réel mais est la conséquence d'une situation politique et militaire très difficile. On passe d'une migration économique à une migration forcée. Arrivés en assez grand nombre pour un pays comme la Suède, ils connaissent des conditions d'accueil que la majorité d'entre eux décrit comme bonnes, malgré des refus d'entrée sur le territoire suédois, injustifiés dans un certain nombre de cas (Assar, 1995). Selon le Bureau de l'immigration suédois les arrivées sont très nombreuses en 1984, date à laquelle de nombreux Palestiniens fuient les hostilités à Beyrouth et au Sud Liban, ou sont directement transférés par la Croix Rouge vers Chypre ou les pays d'Europe du Nord.

Aux côtés de la Suède, on trouve l'Allemagne qui continue d'accueillir des Palestiniens. Cependant il devient de plus en plus difficile pour les réfugiés d'y obtenir le droit à la résidence. Certains décident donc de poursuivre leur migration vers l'Europe du Nord. Le Danemark accueille aussi à l'époque un grand nombre de réfugiés qui y arrivent directement ou qui viennent d'Allemagne.

Figure 1 : Les demandeurs d'asile palestiniens en Suède (1981-1997)

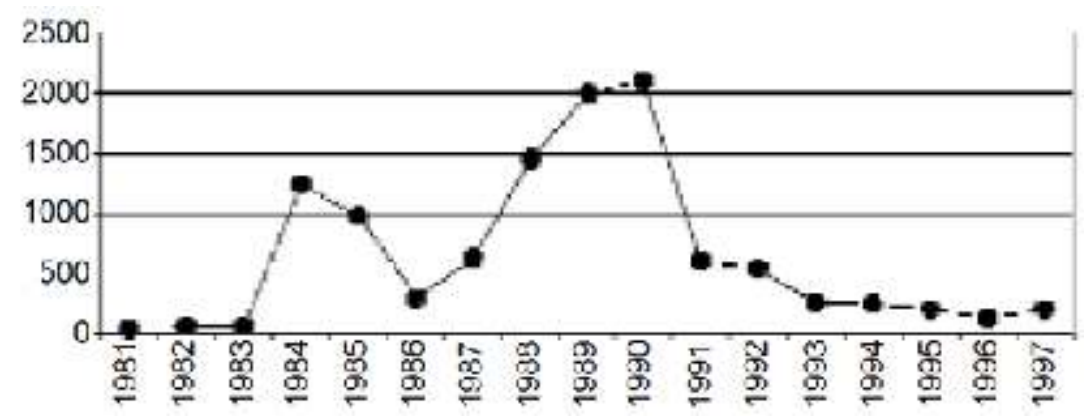

Note : Les entrées représentées dans ce graphique relèvent de la catégorie « apatride » dans les statistiques suèdoises. L'organisme qui produit ces données indique qu'il s'agit de fait des Palestiniens.

Source : Migrationsverker, 2002. 
Figure 2 : Les demandeurs d'asile palestiniens au Danemark (1986-2000)

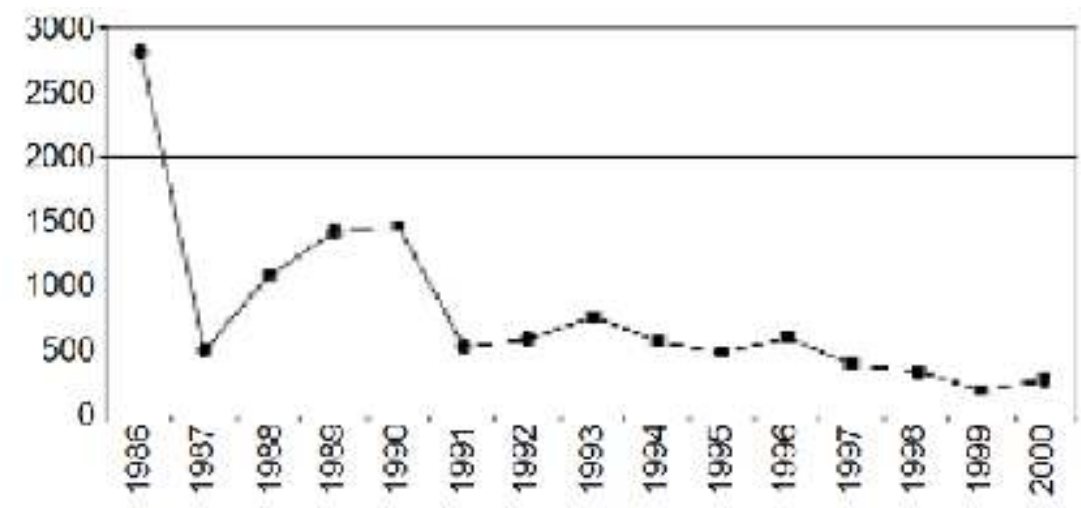

Note : Le Danish Immigration Service utilise la catégorie « Stateless Palestinians » dans ses statistiques.

Source : De 1985 à 1990 : Eurostat $(1994$ : 64, 123); de 1991 à 2000 : Danish Immigration Service (2001).

\section{Le développement des migrations illégales à partir de 1987}

La quatrième phase d'arrivées nombreuses se situe entre 1988 et 1991. Elle fait suite à la guerre des camps qui a opposé les Palestiniens à la milice chiite libanaise Amal. De nombreux camps dont celui de Shatila à Beyrouth, ou celui de Rashidiyyeh à Tyr ont subi un blocus de plusieurs mois, poussant de nombreux Palestiniens à l'exil interne. Un camp informel, Al Sikka, s'est formé à Saïda à proximité du camp de Aïn el Heloueh, regroupant les déplacés internes palestiniens. De nombreuses habitations palestiniennes ont été détruites durant la guerre des camps, et certaines familles ont préféré alors l'exil vers les pays scandinaves au retour à Beyrouth ou Tyr. À l'époque, une filière migratoire s'est constituée entre Saïda d'une part, le Danemark et la Suède d'autre part. Les arrivées de Palestiniens ont diminué à partir de 1991, avec le retour de la paix au Liban, pour augmenter de nouveau après 1993. Des raisons économiques et juridiques expliquent la reprise de l'émigration. La situation économique des Palestiniens s'est dégradée très fortement à partir de 1993, beaucoup de jeunes cherchent donc à partir vers l'Europe pour trouver un emploi. Les Accords d'Oslo ne permettent pas, pour les Palestiniens du Liban, d'envisager l'avenir avec optimisme, certains partent vers l'Europe pour obtenir un statut juridique stable et durable.

\section{Berlin-Est et Chypre, principaux espaces de transit}

La migration des réfugiés palestiniens se fait alors par étapes. Les pays de transit ne sont pas de simples espaces de passage mais sont des lieux de résidence temporaire pouvant aller de quelques mois à quelques années. Le rôle des communautés palestiniennes expatriées déjà présentes sur place est notable à plusieurs égards. L'importance numérique de la communauté détermine le choix des pays de transit. L'Allemagne est le principal pays de transit avec le Danemark, en raison de la forte communauté qui y réside déjà. Les réseaux de solidarité villageois et familiaux y sont activés pour faciliter l'adaptation du migrant à la société d'accueil. Par exemple, les Palestiniens qui passent par l'Allemagne ne parlent pas la langue allemande et ne connaissent pas les administrations qui vont leur permettre d'obtenir des papiers ou 
une aide sociale. La communauté palestinienne d'accueil informe les réfugiés des possibilités de se rendre dans des pays voisins (Suède, Danemark) et facilite leurs déplacements en prenant en charge une partie ou la totalité du coût du voyage. Elle leur permet aussi de trouver du travail de façon temporaire, le plus souvent illégalement, afin de gagner l'argent qui va leur permettre de poursuivre sa migration.

Jusqu'à la fin des années quatre-vingt les Palestiniens du Liban qui voulaient émigrer se rendaient en premier lieu à Damas, la capitale syrienne voisine pour se diriger ensuite vers Berlin. À cette époque un billet d'avion pour la capitale est-allemande ne coûtait que 200 \$ par personne. Les autorités est-allemandes ne demandaient pas de visa, ce qui simplifiait fortement les procédures pour les Palestiniens qui ne disposent pas de passeport mais de simples documents de voyage fournis par leur premier pays d'accueil. Ensuite il fallait passer à l'Ouest et demander le statut de réfugié. Les autorités allemandes ont longtemps accepté l'arrivée de Palestiniens sans les refouler. De ce fait Berlin est devenu un point d'entrée pour l'Europe de l'Ouest. Beaucoup de réfugiés palestiniens ont emprunté cette voie pour ensuite se rendre au Danemark et en Suède ${ }^{3}$.

Jamal, un jeune chauffeur de taxi palestinien à Göteborg est un bon exemple de cette première arrivée de réfugiés palestiniens. Marié à une suédoise, il a deux enfants. Il a obtenu la nationalité suédoise. Blessé pendant la guerre du Liban, son arrivée en Suède est le résultat d'un parcours migratoire complexe :

«J'habitais en Allemagne avant de venir ici. Je venais du Liban. Je suis arrivé après l'invasion israélienne. J'ai été blessé dans la guerre au mois d'août 1982. Je suis parti du Liban avec le CICR ${ }^{4}$. Ils m'ont emmené à Chypre, puis en Grèce. De là je suis parti en RDA. J'y suis resté 20 ou 25 jours avec des membres de l'OLP qui étaient là-bas. J'étais en Allemagne de l'Est, puis ils m'ont emmené à Berlin pour me changer d'hôpital. Un de mes frères travaillait pour l'OLP en RFA. Je l'ai contacté pour qu'il m'aide à retourner en Syrie. Mais mon frère connaissait bien la situation là-bas, et ne voulait pas que j'y aille. Il a tout fait pour me faire passer en RFA. J'y suis arrivé au début de 1983. En règle générale, la situation des réfugiés était difficile. Ils ont refusé que je m'y installe. Je suis resté longtemps en RFA, presque 6 mois, pour soigner ma blessure. En RFA, nous n'avions pas le droit de travailler, ni d'étudier. Nous n'avions pas accès à l'aide sociale. Après ma sortie de l'hôpital, j'ai cherché du travail dans les restaurants. À cette époque c'était la seule profession possible. J'ai travaillé un temps sans permis, pendant un an.

Avec la fin de l'invasion israélienne à Beyrouth j'ai voulu rentrer au Liban. Mais il y avait encore beaucoup de problèmes là-bas. Un de mes frères resté au Liban m'en a dissuadé. Dans l'heure j'ai pris une décision. Je vais aller en Suède, s'ils ne m'acceptent pas, je rentre au Liban malgré ce que m'a dit mon frère. Je suis entré en Suède, et ils m'ont donné le permis de résidence au bout de cinq mois... Pourquoi j'ai choisi la Suède ? À cette époque on parlait beaucoup de la Suède. C'est la seule raison de ma venue. Beaucoup de Palestiniens, et d'autres réfugiés ont trouvé refuge ici ».

Houda, originaire de Shatila à Beyrouth a connu, elle, une longue escale à Chypre. Une femme seule rencontre encore plus de difficultés. Son arrivée en Suède ne résulte pas non plus d'un choix, mais cette destination lui est apparue comme la seule solution envisageable. Le départ est alors comme l'ultime réponse face à l'absence de possibilité, tant de rester à Chypre que de retourner au Liban, ou d'aller dans un autre pays arabe. Elle vit actuellement seule à Göteborg avec sa jeune fille. La présence d'une partie de sa famille à Göteborg explique le choix de sa destination : 
« Je suis partie à Chypre en 1983. J'y suis restée jusqu'en 1991, date à laquelle je suis arrivée en Suède. Entre temps, il y a eu la guerre des camps au Liban. Ma famille (ma mère, mon frère et ma sœur) est venue à ce moment là en Suède. Moi je suis restée à Chypre. Je ne voulais pas partir, je voulais libérer la Palestine, résister. J'étais contre l'émigration. Mais après la guerre du Golfe, il y a eu beaucoup de problèmes avec l'OLP. Les Palestiniens du Liban à l'étranger n'avaient plus aucun avenir devant eux. Je n'avais aucun lieu où retourner étant donnée la situation au Liban. D'autant plus que j'étais enceinte à cette époque. La Suède m'est alors apparue comme la seule solution ».

\section{La complexification des parcours migratoires des Palestiniens du Liban}

L'émigration clandestine s'est développée avec la dégradation de la situation des Palestiniens du Liban, principal foyer d'émigration vers l'Europe dans les années 1990, et la fermeture progressive des frontières européennes aux réfugiés. Les filières migratoires qui s'étaient constituées dans les années soixante-dix, et surtout quatrevingt, se sont réactivées. On assiste cependant à une complexification des parcours migratoires, liée au caractère clandestin de cette émigration.

\section{Le fonctionnement de l'émigration clandestine}

Les Palestiniens de la diaspora n'ont pas de passeports mais disposent de documents de voyages qui leurs sont fournis par l'État libanais. La majeure partie des pays européens ne donnent pas de visa aux Palestiniens, et ne leurs permettent plus d'obtenir l'asile politique ou le statut de réfugié humanitaire. Des grands pays d'accueil, comme l'Allemagne, le Danemark ou la Suède, précédemment étudiés, ont durci leur politique d'accueil envers les Palestiniens. Les réfugiés qui veulent émigrer doivent élaborer des stratégies migratoires qui leur permettent de quitter le territoire libanais et atteindre les pays européens de leur choix, sans passer par une voie légale.

La méthode la plus employée est l'achat d'un billet d'avion pour une destination du tiers-monde, pour lequel les Palestiniens obtiennent un visa de tourisme. Il faut choisir un avion qui fasse escale dans un pays européen. Lors de l'escale, ces derniers demandent l'asile politique. L'organisation de ces filières est entre les mains de passeurs au Liban, qui organisent le voyage et achètent les visas nécessaires; le plus souvent il s'agit de faux visas. Nous avons rencontré un passeur qui nous a décrit son mode de travail :

«Tout d'abord quelqu'un vient te voir qui veut voyager. Il te donne son passeport et 1500 \$. Les passeurs sont des gens qui travaillent dans le voyage. Le passeur te donne un visa et un billet d'avion pour que tu partes. Tu arrives à partir ou non, en fonction de ta chance. Si tu ne pars pas, il garde les 1500 \$ pour le travail qu'il a fourni, le visa et le billet.

Il faut avoir des relations avec beaucoup de monde, tout d'abord avec les gens à la douane ici. Il faut aussi connaître des gens importants à Beyrouth qui te permettent d'avoir des visas. Une fois que tu as eu le visa et le billet, celui qui veut partir doit payer en plus. Le prix dépend du passeur, ainsi que les modalités de paiement, ça dépend. Les passeurs connaissent des gens ici qui travaillent pour l'État libanais. On travaille avec des passeurs plus importants, c'est eux qui ont des relations avec les gens dans les ambassades et à l'étranger. 
Nous sommes aussi en contact avec des gens à l'extérieur du Liban. Maintenant on arrive à avoir des visas pour les pays d'Afrique, et des pays comme l'Uruguay, l'Argentine, des pays comme ça... Tout vient de l'extérieur.

Pour l'Allemagne cela coûte 4500 \$ pour un Palestinien, 4000 \$ pour un Libanais, c'est toujours plus cher pour un Palestinien. Ici un passeur gagne au moins $500 \$$ par individu qui part. Il peut gagner plus $»$.

31 Les filières, très lucratives pour les passeurs, sont de plus en plus difficiles à élaborer. $\mathrm{Au}$ fur et à mesure de leur découverte par les autorités libanaises, elles sont démantelées. Les filières et les parcours migratoires se complexifient et comportent de plus en plus d'étapes pour brouiller les pistes.

\section{La multiplication des étapes migratoires}

Le passeur que nous avons rencontré poursuit son explication et nous décrit la grande complexité des parcours migratoires qu'il faut mettre en place :

« Pour l'Angleterre il faut avoir deux billets d'avion. D'abord tu pars en Thaïlande, puis de là-bas tu reviens vers Beyrouth avec une escale à Londres. Si ça marche c'est bon, sinon tu reviens ici. On ne peut pas faire plus ».

Les parcours migratoires des Palestiniens du Liban touchent des espaces de plus en plus vastes, recouvrant pour partie l'extension de la diaspora libanaise qui peut servir dans certains cas de relais dans les pays de transit. Nous avons pu faire ces observations lors d'entretiens au Sud Liban avec des personnes qui ont tenté l'émigration et d'autres informateurs, comme des passeurs. Les principaux espaces de transit que nous avons relevés sont des pays d'Afrique de l'Ouest, d'Amérique latine ou d'Asie (carte). Des billets retour vers Beyrouth via un pays européen sont pris à partir des capitales de ces pays.

Carte : Quelques exemples de diversification des parcours migratoires des Palestiniens du Liban : principaux pays de transit et d'accueil

Khalil, un jeune palestinien du camp d'Al Buss raconte son histoire, qui s'est soldée quatre fois par des échecs :

« Il n'y avait pas de travail. On entendait parler les gens qui étaient partis, que la situation était meilleure à l'étranger quand on est Palestinien. Et puis il y a la question de la nationalité... Les nationalités européennes simplifient beaucoup la vie. Nous avons été voir un passeur, nous lui avons payé 4000 \$, il voulait 5000 \$... On lui en a donné 2000 tout de suite et on payerait le reste en arrivant. Nous étions sept amis en tout.

Après un mois, il nous a dit que l'on pouvait partir. C'était en 1997. On est parti par l'aéroport de Beyrouth. La première chose qui est arrivée quand on a passé la douane, ils nous ont mis de côté parce qu'on est Palestiniens. Ils nous ont fouillé partout. Nous avons dit que nous partions juste en vacances, mais pour eux un Palestinien qui part en vacances ce n'est pas possible. Nous partions pour devenir des migrants illégaux, mais nos visas et nos passeports au départ étaient en règle. À la fin ils nous ont laissé partir.

Nous sommes partis pour Conakry. On est descendu-là et on a rencontré un type, qui devait nous emmener vers Londres. Nous sommes restés à Conakry et nous avons arrangé les billets et les visas pour passer en transit par Londres. Nous avons eu un billet pour Beyrouth via Bruxelles et Londres. Nous sommes partis d'Afrique pour l'Europe.

C'était des passeurs libanais en Afrique. Nous sommes arrivés à Bruxelles. On est sorti ensemble, à sept, et on a demandé l'asile. Ils nous ont gardé à l'aéroport huit 
heures puis on a été à la police. On est resté là 21 jours. On a demandé l'asile politique. Au bout de quinze jours on a eu un premier refus. On a demandé une deuxième fois, on a eu un autre refus. Après deux refus, khalas... Je me suis blessé les bras avec un couteau, il y avait du sang partout... Mon cousin aussi a fait pareil. Nous voulions coûte que coûte entrer dans le pays, comme ça ils nous ont emmenés dans un hôpital. Ça n'a pas fonctionné. Nous sommes revenus au Liban.

En arrivant ils nous ont confisqué nos passeports après nous avoir retenus deux ou trois heures. Nous sommes partis de l'aéroport et nous sommes revenus dans le camp. Après un certain temps, j'ai été récupérer mon passeport et j'ai tenté de nouveau de partir. Je suis repassé par le même passeur. Je suis ensuite reparti pour passer par l'Algérie. En Algérie j'avais un billet pour La Havane, via Londres avec un retour. Tu vois... Ou à l'aller, ou au retour, je demandais l'asile à Londres ».

Malgré ces échecs ce jeune Palestinien, compte toujours essayer de partir pour l'Europe avec ses amis.

\section{Les réseaux familiaux et villageois : les ressources de l'exilé} tant dans le pays de départ que dans le pays d'accueil. Les sommes investies dans les voyages et le paiement des nombreux intermédiaires sont comprises entre 4000 et 7 $000 \$$ et dépendent des destinations. Lors de nos entretiens au Sud Liban nous avons rencontré des familles entières qui ont tenté de partir, ce qui place les sommes investies, et perdues pour ceux qui ont connu l'échec, à plus de 15000 \$ dans certains cas. Plusieurs familles rencontrées, entre autre dans le camp de Borj Shémali, le plus défavorisé de la région de Tyr, ont vendu tous leurs biens, dont leur habitation et leurs meubles pour partir. Un échec les met dans une situation socio-économique très difficile. Les personnes qui désirent émigrer empruntent le plus souvent leur argent dans les réseaux familiaux et villageois. Khalil témoigne de la façon dont il a récolté l'argent nécessaire :

«C'est avec des prêts, auprès de mes proches, de ma sœur, de mes amis, c'est comme ça que j'ai ramassé la somme ».

Dans le pays d'arrivée, les proches ainsi que des personnes du même village d'origine en Palestine ou du même camp de réfugiés jouent un rôle important, comme dans le cas de Jamal à Göteborg. Ce sont ces réseaux qui permettent de trouver un emploi, ou un logement dès l'arrivée.

\section{Conclusion}

La complexification des parcours migratoires des réfugiés palestiniens qui quittent leur pays de résidence pour tenter de se rendre en Europe répond à la multiplication des barrières juridiques qui se dressent devant eux, tant dans leur pays de départ que dans les espaces de transit ou de destination finale. Il s'ensuit donc le développement d'une migration clandestine, très coûteuse pour les candidats au départ, qui s'appuie sur les réseaux migratoires formés depuis les années soixante-dix et surtout quatre-vingt par les premiers arrivants palestiniens. Il est à l'heure actuelle très difficile de spécifier de quel type de migration il s'agit, tant les facteurs qui en sont à l'origine sont divers et imbriqués. D'un côté, ces longs et complexes parcours migratoires font penser à ceux des migrants clandestins qui viennent en Europe pour travailler, d'un autre, leur statut juridique particulier et le contexte politique actuel au Moyen-Orient les rapprochent de 
ces réfugiés pour qui le retour dans leur pays d'origine, la Palestine, est interdit et dont la présence dans certains pays d'accueil est de plus en plus remise en cause. Migration forcée, recherche d'asile et migration économique sont les trois facettes de la réalité migratoire des Palestiniens d'Europe. L'Europe demeure cependant un espace assez marginal au sein de la diaspora palestinienne, et le faible degré d'organisation des réfugiés qui y résident, ainsi que le manque d'institutions diasporiques palestiniennes sur ce continent la différencient des Palestiniens des États-Unis, qui forment un pôle organisé et structuré. Seuls les Palestiniens du Royaume-Uni tentent de promouvoir un certain nombre d'organisations palestiniennes dont le but est la défense du droit au retour. Ce type de démarche reste toutefois embryonnaire et ne fédère pas l'ensemble des Palestiniens du Vieux continent.

\section{BIBLIOGRAPHIE}

AL-NATOUR Souheil (1997) The legal status of Palestinians in Lebanon, Journal of Refugee Studies (Special Issue : Palestinians in Lebanon), vol. 10, $\mathrm{n}^{\circ}$ 3, sept., pp. 360-377.

ASSAR Selma (1995) The Palestinian Refugees. Analysis and Comparison of Two Recipient Countries Behaviour : Sweden and Jordan - 1948-1991 -, Högskolan i Vaxjö, Institute of Social Sciences (non publié).

DESTREMAU Blandine (1993) Le Statut juridique des Palestiniens vivant au Proche-Orient, Revue d'Études Palestiniennes, $n^{\circ}$ 98, pp 35-62.

DORAÏ Mohamed Kamel (2000) Les parcours migratoires des Palestiniens vers la Suède et l'Europe du Nord, Revue d'Études Palestiniennes, nº 23, nouvelle série, pp. 38-52.

Guillon Michelle, Legoux Luc, Ma Mung Emmanuel éds, L'asile politique entre deux chaises, Droits de l'Homme et gestion des flux migratoires, L'Harmattan, coll Espaces interculturels, 377 p.

ISSA Mahmoud (1995) Palestinians from Lubya in Denmark. Dreams and realities, working paper, Danish Refugee Council, The Documentation Centre, Copenhagen, 80 p.

RAZQALLAH Hala Nawfal (1998) Les Palestiniens au Liban et en Syrie, une étude comparée (1948-1995), Dar al jadid, Beyrouth, 111 p. (en arabe).

\section{NOTES}

1. Une exception est à noter: les Palestiniens sont exemptés de montrer leur document de voyage à la frontière entre le Liban et la Syrie (Décret 1188, 1967).

2. L'ensemble des noms cités dans cette étude sont des pseudonymes pour préserver l'anonymat des personnes interrogées.

3. Cette filière nous a été décrite par Abdel Qader Yassine (chercheur à l'Université de Göteborg) à Borås (août 1998).

4. Comité International de la Croix Rouge. 


\section{RÉSUMÉS}

Quelques 100000 Palestiniens vivent en Europe. Le continent leur est apparu à partir des années soixante-dix comme un espace favorable leur permettant de trouver du travail et d'assurer à leur famille une vie plus stable. L'Europe peut-elle devenir dans l'avenir une région importante au sein de la diaspora palestinienne ? S'agit-il d'une région de réinstallation des Palestiniens ou bien d'un espace d'émigration de travail comme les pays producteurs de pétrole ? Peut-on imaginer une évolution équivalente à celle des Palestiniens des États-Unis qui forment un groupe dynamique et structuré au sein de la diaspora?

Après un panorama général des principales communautés palestiniennes en Europe, la situation juridique des Palestiniens du Liban, principale cause de départ de cette communauté, est analysée ; puis les principales phases d'arrivée en Europe sont distinguées pour ensuite analyser le fonctionnement actuel de la migration clandestine. Le Liban, qui représente le principal pays de départ vers l'Europe, est étudié plus en détail. La situation en Suède est, elle aussi, analysée plus amplement du fait des spécificités de ce pays d'accueil et la relative méconnaissance de la situation des Palestiniens qui y résident. Ce travail se fonde essentiellement sur deux études de terrain au Liban et en Suède.

\section{Palestinian Refugees in Europe. A Complexity of Migratory Routes and Spaces}

Some 100000 Palestinians live in Europe. The continent began to appear a favourable place for Palestinians in the1970's, allowing them to find work and ensure a stable family life. Might Europe become an important region for the Palestinian Diaspora in the future? Is it a region of resettlement for Palestinians or a space for work emigration like the petroleum producing countries? Might we foresee an evolution similar to that of the Palestinians of the United States who form a dynamic and structured group within the Diaspora?

After a general overview of the main Palestinian communities in Europe and of the legal situation of the Palestinians of Lebanon, the main causes of departure of this community are analysed. The phases of arrival in Europe are differentiated to then analyse the current workings of clandestine migration. Lebanon, which represents the main country of departure toward Europe, is studied in more detail. The situation of Sweden is also further analysed due to the specificity of this host country and the relative lack of knowledge of the situation of the Palestinians who live there. This work is based essentially on two field studies in Lebanon and Sweden.

Los refugiados palestinos en Europa. Complejidad de de los recorridos y de los espacios migratorios

Alrededor de 100000 palestinos viven actualmente en Europa. La comunidad palestina considera, desde los anos setenta, que el continente europeo constituye un espacio favorable puesto que ofrece, a aquellos que deciden instalarse, un trabajo y una vida familiar más estable. ¿ Puede Europa, en un futuro, convertirse en una región importante par la diáspora palestina ? ¿ Se trata, para los palestinos, de una verdadera región de reinstalación o más bien de un espacio de inmigración por cuestiones de trabajo siguiendo el ejemplo de los países productores de petróleo ? ¿Podemos imaginar una evolución equivalente a la de los palestinos instalados en Estados Unidos ; es decir, la de un grupo dinámico y estructurado en el seno de la diáspora?

Este artículo presenta, en primer lugar, un panorama general de las principales comunidades palestinas de Europa analizando el motivo principal de la partida : la situación jurídica de los palestinos instalados en el Líbano. En segundo lugar, el autor diferencia las sucesivas fases de la llegada de palestinos a Europa para, a continuación, estudiar el funcionamiento actual de la inmigración clandestina. Por último, el autor analiza el caso sueco en virtud de los particularismos de este país de acogida y del relativo desconocimiento de la situación de los 
palestinos en él instalados. Este trabajo se basa, esencialmente, en dos estudios de terreno realizados en el Líbano y en Suecia.

INDEX

Mots-clés : flux, Palestiniens, processus migratoire

Index géographique : Europe, Liban, Suède

\section{AUTEUR}

MOHAMED KAMEL DORAÏ

Chargé de recherche CNRS, équipe Migrinter (Unité mixte de recherche 6588 CNRS, Maison des Sciences de l'Homme et de la Société, 99 av du Recteur Pineau, Poitiers, France. Courriel :

Mohamed-Kamel.Dorai@univ-poitiers.fr 